UUITP-12/07

NORDITA-2007-22

YITP-SB-07-22

\title{
T-duality and Generalized Kähler Geometry
}

\author{
Ulf Lindström ${ }^{a, b, c}$, Martin Roček ${ }^{d}$, Itai Ryb ${ }^{d}$, \\ Rikard von Unge ${ }^{e}$, and Maxim Zabzine ${ }^{a}$ \\ ${ }^{a}$ Department of Theoretical Physics Uppsala University, \\ Box 803, SE-751 08 Uppsala, Sweden \\ ${ }^{b}$ HIP-Helsinki Institute of Physics, University of Helsinki, \\ P.O. Box 64 FIN-00014 Suomi-Finland \\ ${ }^{c}$ NORDITA, Roslagstullsbacken 23, \\ SE-10691 Stockholm, Sweden \\ ${ }^{d}$ C.N.Yang Institute for Theoretical Physics, Stony Brook University, \\ Stony Brook, NY 11794-3840,USA \\ ${ }^{e}$ Institute for Theoretical Physics, Masaryk University, \\ 61137 Brno, Czech Republic
}

\begin{abstract}
We use the new $N=(2,2)$ vector multiplets to clarify T-dualities for generalized Kähler geometries. Following the usual procedure, we gauge isometries of nonlinear $\sigma$-models and introduce Lagrange multipliers that constrain the field-strengths of the gauge fields to vanish. Integrating out the Lagrange multipliers leads to the original action, whereas integrating out the vector multiplets gives the dual action. The description is given both in $N=(2,2)$ and $N=(1,1)$ superspace.
\end{abstract}




\section{Introduction}

The basic inspiration for our work is the interesting duality found in [1, 2] for two dimensional nonlinear $\sigma$-models with $N=(2,2)$ supersymmetry and target space geometries that are not Kähler. As was shown in [3, 4, T-dualities arise when one gauges an isometry, and then constrains the field-strength of the corresponding gauge field to vanish. In this paper, we use the new vector multiplets introduced in [5, 6] to describe T-duality for generalized Kähler geometries (for a sampling of articles in the field, see [7]). We first work in $N=(2,2)$ superspace, and then reduce to $N=(1,1)$ superspace and find the usual T-duality of Buscher [8].

The plan of the paper is as follows: In the next section we briefly review T-duality in the pure Kähler case [8]. We then review the classes of isometries that generalized Kähler geometries admit. Next, we consider T-dualities along isometries in the kernel of the commutator of the left and right complex structures that mix chiral and twisted chiral multiplets [9]. Finally we describe T-dualities along isometries in the cokernel of the commutator, which act only on the semichiral multiplets[10]. We use the bihermitian description of generalized Kähler geometry throughout the paper, and leave the description of T-duality in terms of generalized complex structures to the future.

We end with a brief conclusion.

\section{Kähler geometry and T-duality}

In this section, we briefly review isometries, gauging, and T-duality in $N=(2,2)$ and $N=(1,1)$ superspace for a system with chiral superfields $\phi^{a}$ and an $N=(2,2)$ superspace Lagrange density given by a Kähler potential $K\left(\phi^{a}, \bar{\phi}^{a}\right)$ [11, 4, 8]. For simplicity, we consider an isometry generated by a holomorphic Killing vector $k$ that leaves the Kähler potential invariant 1

$$
k \equiv k^{i} \partial_{i}=k^{a} \partial_{a}+\bar{k}^{a} \bar{\partial}_{a} \quad, \quad \mathcal{L}_{k} K=0
$$

where $\varphi^{i}=\left\{\phi^{a}, \bar{\phi}^{a}\right\}$. The isometry is gauged using a multiplet $V^{\phi}$ to promote the constant (real) transformation parameter $\lambda$ to a complex chiral superfields $\Lambda$ :

$$
\lambda\left(k^{a} \partial_{a}+\bar{k}^{a} \bar{\partial}_{a}\right) K\left(\phi^{a}, \bar{\phi}^{a}\right)=0 \rightarrow\left(\Lambda k^{a} \partial_{a}+\bar{\Lambda} \bar{k}^{a} \bar{\partial}_{a}+\delta V^{\phi} \partial_{V^{\phi}}\right) K^{(g)}\left(\phi^{a}, \bar{\phi}^{a}, V^{\phi}\right)=0 .
$$

\footnotetext{
${ }^{1}$ The general case when $K$ is invariant only up to a Kähler transformation is discussed in detail in [11, and in the generalized Kähler case in [12].
} 
From (2.1), it follows that 2

$$
\left(\Lambda k^{a} \partial_{a}+\bar{\Lambda} \bar{k}^{a} \bar{\partial}_{a}\right) K\left(\phi^{a}, \bar{\phi}^{a}\right)=\frac{i}{2}(\bar{\Lambda}-\Lambda) \mathcal{L}_{J k} K \quad, \quad J k=i\left(k^{a} \partial_{a}-\bar{k}^{a} \bar{\partial}_{a}\right)
$$

Using the usual gauge transformation $\delta V^{\phi}=i(\bar{\Lambda}-\Lambda)$, we find the gauged action [11]:

$$
K^{(g)}\left(\phi^{a}, \bar{\phi}^{a}, V^{\phi}\right)=\exp \left(-\frac{1}{2} V \mathcal{L}_{J k}\right) K\left(\phi^{a}, \bar{\phi}^{a}\right) .
$$

To find the T-dual model [4], we constrain the twisted chiral field-strength $\overline{\mathbb{D}}_{+} \mathbb{D}_{-} V^{\phi}$ to vanish. We impose this with a Legandre transformation of the density with a twisted chiral Lagrange multiplier $\chi$ :

$$
K^{(g)}\left(\phi^{a}, \bar{\phi}^{a}, V^{\phi}\right)-(\chi+\bar{\chi}) V^{\phi}
$$

In $N=(2,2)$ superspace, we find the T-dual Lagrange densities by integrating out either $\chi+\bar{\chi}$, which gives the original Kähler potential, or $V^{\phi}$, which gives the T-dual potential $\tilde{K}\left(\chi+\bar{\chi}, x^{A}\right)$ where $x^{A}$ are "spectator" fields, i.e., combinations of the $\varphi^{i}$ that are inert under the action of the isometry (2.1). The geometric nature of the duality is made manifest when we descend to $N=(1,1)$ superspace. In Wess-Zumino gauge, the $N=(1,1)$ components of the multiplet $V^{\phi}$ and the covariant derivatives are

$$
V^{\phi}\left|=0 \quad, \quad Q_{ \pm} V^{\phi}\right|=A_{ \pm} \quad, \quad i Q_{+} Q_{-} V^{\phi} \mid=d, \quad \nabla_{ \pm} \varphi^{i}=D_{ \pm} \varphi^{i}-A_{ \pm} k^{i}
$$

the constrained Lagrange density (2.5) becomes:

$$
g_{i j} \nabla_{+} \varphi^{i} \nabla_{-} \varphi^{j}-i d\left(K_{i} J_{j}^{i} k^{j}+(\chi+\bar{\chi})\right)+f(\chi-\bar{\chi})
$$

where $f=i\left(D_{+} A_{-}+D_{-} A_{+}\right)$is the $N=(1,1)$ field-strength for the gauge fields, $g_{i j}$ is the Kähler metric, and $K_{i} J_{j}^{i} k^{j} \equiv \mathcal{L}_{J k} K$ is proportional to the moment map when the Kähler potential is invariant (as discussed above, in general $\mathcal{L}_{J k} K \rightarrow-\mu$ ). Integrating out the $N=(1,1)$ auxiliary superfield $d$ sets $\chi+\bar{\chi}$ equal to the moment map. This can be solved either by expressing $\chi+\bar{\chi}$ as a function of $\varphi^{i}$, or by changing coordinates to $\chi+\bar{\chi}$ and a combination of $\varphi^{i}$ algebraically independent of the moment map; the two procedures are related simply by a diffeomorphism. This gives the $N=(1,1)$ gauged Lagrange density with Lagrange multiplier $(\chi-\bar{\chi})$ constraining the field-strength $f$ :

$$
\mathcal{L}_{1}=g_{i j} \nabla_{+} \varphi^{i} \nabla_{-} \varphi^{j}+f(\chi-\bar{\chi}),
$$

where $g_{i j}$ is the original Kähler metric (in $\varphi^{i}$ coordinates). Thus $N=(2,2)$ T-duality is the same as $N=(1,1)$ T-duality up to an accompanying diffeomorphism; this was originally proven by Buscher [8, but not explicitly spelled out.

\footnotetext{
${ }^{2}$ If $K$ is not invariant, in (2.3) $\mathcal{L}_{J k} K$ must be replaced by the moment map of $k: \mathcal{L}_{J k} K \rightarrow-\mu$.
} 


\section{$3 \quad$ T-duality for the generalized Kähler geometry}

In a recent paper [5] we discussed gauge multiplets suitable for gauging isometries of generalized Kähler geometries. We found three distinct vector multiplets, corresponding to three distinct types of isometries: those along the kernel of either $J_{+}-J_{-}$or (equivalently) $J_{+}+J_{-}$, those acting on both kernels, and those along the cokernel of the commutator $\left[J_{+}, J_{-}\right]$. The isometries can be expressed, following [1], in adapted coordinates:

$$
\begin{aligned}
k_{\phi} & =i\left(\partial_{\phi}-\partial_{\bar{\phi}}\right), \\
k_{\phi \chi} & =i\left(\partial_{\phi}-\partial_{\bar{\phi}}-\partial_{\chi}+\partial_{\bar{\chi}}\right), \\
k_{L R} & =i\left(\partial_{L}-\partial_{\bar{L}}-\partial_{R}+\partial_{\bar{R}}\right) .
\end{aligned}
$$

If we assume that the generalized Kähler potential is invariant, the corresponding gauged actions are:

$$
\begin{aligned}
K_{\phi} & =K_{\phi}\left(\phi+\bar{\phi}+V^{\phi}, x\right) \\
K_{\phi \chi} & =K_{\phi \chi}\left(\phi+\bar{\phi}+V^{\phi}, \chi+\bar{\chi}+V^{\chi}, i(\phi-\bar{\phi}+\chi-\bar{\chi})+V^{\prime}, x\right), \\
K_{\mathbb{X}} & =K_{\mathbb{X}}\left(\mathbb{X}_{L}+\overline{\mathbb{X}}_{L}+\mathbb{V}^{L}, \mathbb{X}_{R}+\overline{\mathbb{X}}_{R}+\mathbb{V}^{R}, i\left(\mathbb{X}_{L}-\overline{\mathbb{X}}_{L}+\mathbb{X}_{R}-\overline{\mathbb{X}}_{R}\right)+\mathbb{V}^{\prime}, x\right),
\end{aligned}
$$

where $x$ represents all possible spectator fields. The case (3.4) is essentially identical to the Kähler case above; aside from subtleties pertaining to the interpretation of the moment map, which will be discussed in [12], there are no new features. We now consider (3.513.6) in detail, and show that they again reduce to standard Buscher duality in $N=(1,1)$ superspace, along with some natural diffeomorphisms inherited from $N=(2,2)$ superspace. A more general discussion of isometries and moment maps will be given in [12].

\subsection{T-duality for an isometry $k_{\phi \chi}$}

For an invariant generalized Kähler potential $K$ in adapted coordinates, the gauged action is (3.5). In the special circumstance when all the spectators are (twisted) chiral, we can give a nice geometric interpretation of the gauging analogous to the Kähler case above. In this case both complex structures are simultaneously diagonalizable; and the manifold has the Bihermitian Local Product (BiLP) geometry defined in [13]. Using the invariance of $K$ under $k_{\phi \chi}$, and using the complex structures $J_{ \pm}$and their product $\Pi=J_{+} J_{-}$

$$
\begin{aligned}
{\left[i \left(\Lambda \partial_{\phi}\right.\right.} & \left.\left.-\tilde{\Lambda} \partial_{\chi}\right)+ \text { c.c. }\right] K \\
& =\frac{i}{4}\left[(\bar{\Lambda}-\Lambda) \mathcal{L}_{\left(J_{+}+J_{-}\right) k}+(\overline{\tilde{\Lambda}}-\tilde{\Lambda}) \mathcal{L}_{\left(J_{+}-J_{-}\right) k}+i(\Lambda+\bar{\Lambda}-\tilde{\Lambda}-\overline{\tilde{\Lambda}}) \mathcal{L}_{\Pi k}\right] K
\end{aligned}
$$


To gauge the isometry, we require

$$
\begin{aligned}
0= & \delta V^{\alpha} \partial_{V^{\alpha}} K^{(g)} \\
& +\frac{i}{4}\left[(\bar{\Lambda}-\Lambda) \mathcal{L}_{\left(J_{+}+J_{-}\right) k}+(\overline{\tilde{\Lambda}}-\tilde{\Lambda}) \mathcal{L}_{\left(J_{+}-J_{-}\right) k}+i(\Lambda+\bar{\Lambda}-\tilde{\Lambda}-\overline{\tilde{\Lambda}}) \mathcal{L}_{\Pi k}\right] K^{(g)}
\end{aligned}
$$

The three superfields of the large vector multiplet [5] have the right gauge transformations to gauge this symmetry: 3

$$
\begin{gathered}
\delta V^{\phi}=i(\bar{\Lambda}-\Lambda) \quad, \quad \delta V^{\chi}=i(\overline{\tilde{\Lambda}}-\tilde{\Lambda}) \quad, \quad \delta V^{\prime}=(-\Lambda-\bar{\Lambda}+\tilde{\Lambda}+\overline{\tilde{\Lambda}}) \\
\Rightarrow K^{(g)}=\exp \left(-\frac{1}{4} V^{\phi} \mathcal{L}_{\left(J_{+}+J_{-}\right) k}-\frac{1}{4} V^{\chi} \mathcal{L}_{\left(J_{+}-J_{-}\right) k}-\frac{1}{4} V^{\prime} \mathcal{L}_{\Pi k}\right) K .
\end{gathered}
$$

To find the T-dual, we introduce Lagrange multipliers that constrain the field strengths of the large vector multiplet to vanish. As discussed in [5], it is useful to introduce complex potentials for the field-strengths:

$$
\begin{aligned}
& V_{L}=\frac{1}{2}\left[-V^{\prime}+i\left(V^{\phi}-V^{\chi}\right)\right] \Rightarrow \delta_{g} V_{L}=\Lambda-\tilde{\Lambda}, \\
& V_{R}=\frac{1}{2}\left[-V^{\prime}+i\left(V^{\phi}+V^{\chi}\right)\right] \Rightarrow \delta_{g} V_{R}=\Lambda-\tilde{\tilde{\Lambda}} .
\end{aligned}
$$

Since $(\tilde{\Lambda}) \Lambda$ are respectively (twisted)chiral, these give the following gauge invariant complex spinor, semichiral, field-strengths:

$$
\begin{array}{ll}
\mathbb{G}_{+}=\overline{\mathbb{D}}_{+} V_{L}, & \overline{\mathbb{G}}_{+}=\mathbb{D}_{+} \bar{V}_{L}, \\
\mathbb{G}_{-}=\overline{\mathbb{D}}_{-} V_{R}, & \overline{\mathbb{G}}_{-}=\mathbb{D}_{-} \bar{V}_{R} .
\end{array}
$$

Using the chirality properties of the field-strengths we obtain the constrained $N=(2,2)$ generalized Kähler potential, as in (2.5), using semichiral Lagrange multipliers $\tilde{\mathbb{X}}$ :

$$
K^{(g)}-\mathcal{L}_{\text {const. }}=K^{(g)}-\frac{1}{2} \tilde{\mathbb{X}}_{L} V_{L}-\frac{1}{2} \overline{\tilde{\mathbb{X}}}_{L} \bar{V}_{L}-\frac{1}{2} \tilde{\mathbb{X}}_{R} V_{R}-\frac{1}{2} \overline{\tilde{\mathbb{X}}}_{R} \bar{V}_{R} .
$$

This applies to the general case, not just BiLP geometries, though in general, we do not have a nice geometric form of $K^{(g)}$ (this will be discussed in [12]).

\subsubsection{Reduction to $N=(1,1)$ superspace}

Using the results of [5] (as summarized and clarified in Appendix [A), we obtain the $N=(1,1)$ reduction of this action in the Wess-Zumino gauge; the part from $K^{(g)}$ is

$$
\begin{aligned}
\mathcal{L}= & \left(\Xi_{+}^{A}+\nabla_{+} \varphi^{i} E_{i C} E^{C A}\right) E_{A B}\left(\Xi_{-}^{B}+E^{B D} E_{D j} \nabla_{-} \varphi^{j}\right) \\
& +\nabla_{+} \varphi^{i}\left(E_{i j}-E_{i A} E^{A B} E_{B j}\right) \nabla_{-} \varphi^{j} \\
& +i K_{i} k^{j}\left(\hat{q}^{\phi}\left(J_{+j}^{i}+J_{-j}^{i}\right)+\hat{q}^{\chi}\left(J_{+j}^{i}-J_{-j}^{i}\right)+\hat{q}^{\prime} \Pi^{i}{ }_{j}\right)
\end{aligned}
$$

\footnotetext{
${ }^{3}$ Our conventions here, which are compatible with the inherent geometric objects $J_{ \pm}, k$, are slightly different than those introduced in [5]; see Appendix B for the relation between the conventions.
} 
where we introduce the matrices:

$$
\begin{aligned}
& E_{k l}=K_{i j}\left(J_{+k}^{i} J_{-l}^{j}-\frac{1}{2} \Pi_{k}^{i} \delta^{j}{ }_{l}-\frac{1}{2} \Pi^{j}{ }_{l} \delta^{i}{ }_{k}\right) \\
& E_{A l}=K_{i j} k^{k}\left(\begin{array}{c}
J_{-k}^{i} J_{-l}^{j} \\
\Pi_{k}^{i} J_{-l}^{j}
\end{array}\right) \\
& E_{k A}=K_{i j} k^{l}\left(J_{+k}^{i} J_{+l}^{j}, J_{+k}^{i} \Pi^{j}{ }_{l}\right) \\
& E_{A B}=K_{i j} k^{k} k^{l}\left(\begin{array}{cc}
J_{-k}^{i} J_{+l}^{j} & J_{-k}^{i} \Pi^{j}{ }_{l} \\
\Pi^{i}{ }_{k} J_{+l}^{j} & \Pi^{i}{ }_{k} \Pi^{j}{ }_{l}
\end{array}\right)
\end{aligned}
$$

where the normalizations of the auxiliary fields $\Xi_{ \pm}, \hat{q}$ as well as the field-strength $f$ are given in Appendix A.

The constraint reduces to

$$
\begin{aligned}
\mathcal{L}_{\text {const. }} & =\tilde{X}_{L}\left(i \hat{q}^{\prime}-\frac{i}{2} f+\hat{q}^{\phi}-\hat{q}^{\chi}-i D_{+} \Xi_{-}^{2}\right)+\tilde{\psi}_{-}\left(+i \Xi_{+}^{1}-\Xi_{+}^{2}\right) \\
& +\overline{\tilde{X}}_{L}\left(i \hat{q}^{\prime}-\frac{i}{2} f-\hat{q}^{\phi}+\hat{q}^{\chi}+i D_{+} \Xi_{-}^{2}\right)+\overline{\tilde{\psi}}_{-}\left(-i \Xi_{+}^{1}-\Xi_{+}^{2}\right) \\
& +\tilde{X}_{R}\left(i \hat{q}^{\prime}+\frac{i}{2} f+\hat{q}^{\phi}+\hat{q}^{\chi}+i D_{-} \Xi_{+}^{2}\right)+\tilde{\psi}_{+}\left(-i \Xi_{-}^{1}+\Xi_{-}^{2}\right) \\
& +\overline{\tilde{X}}_{R}\left(i \hat{q}^{\prime}+\frac{i}{2} f-\hat{q}^{\phi}-\hat{q}^{\chi}-i D_{-} \Xi_{+}^{2}\right)+\overline{\tilde{\psi}}_{+}\left(+i \Xi_{-}^{1}+\Xi_{-}^{2}\right)
\end{aligned}
$$

where $\tilde{X}=\tilde{\mathbb{X}}\left|, \tilde{\psi}_{+}=Q_{+} \tilde{\mathbb{X}}_{L}\right|$ and $\tilde{\psi}_{-}=Q_{-} \tilde{\mathbb{X}}_{R} \mid$ are the $N=(1,1)$ components of the Lagrange multipliers $\tilde{\mathbb{X}}$.

\subsubsection{T-duality for the large vector multiplet in $N=(1,1)$ superspace}

Integrating out the auxiliaries $\tilde{\psi}_{ \pm}$simply constrains $\Xi_{ \pm}^{A}$ to vanish, and we obtain the gauged Lagrange density:

$$
\begin{aligned}
\mathcal{L} & =K_{i j}\left(J_{+k}^{i} J_{-l}^{j}-\frac{1}{2} \Pi_{k}^{i} \delta^{j}{ }_{l}-\frac{1}{2} \delta^{i}{ }_{k} \Pi_{j}{ }^{l}\right) \nabla_{+} \varphi^{k} \nabla_{-} \varphi^{l} \\
& +i \hat{q}^{\phi}\left(K_{i}\left(J_{+j}^{i}+J_{-j}^{i}\right) k^{j}+i\left(\tilde{X}_{L}-\overline{\tilde{X}}_{L}+\tilde{X}_{R}-\overline{\tilde{X}}_{R}\right)\right) \\
& +i \hat{q}^{\chi}\left(K_{i}\left(J_{+j}^{i}-J_{-j}^{i}\right) k^{j}-i\left(\tilde{X}_{L}-\overline{\tilde{X}}_{L}-\tilde{X}_{R}+\overline{\tilde{X}}_{R}\right)\right) \\
& +i \hat{q}^{\prime}\left(K_{i} \Pi_{j}^{i} k^{j}-\left(\tilde{X}_{L}+\overline{\tilde{X}}_{L}+\tilde{X}_{R}+\overline{\tilde{X}}_{R}\right)\right) \\
& \left.+\frac{i}{2} f\left(\tilde{X}_{L}+\overline{\tilde{X}}_{L}-\tilde{X}_{R}-\overline{\tilde{X}}_{R}\right)\right)
\end{aligned}
$$

Imposing the equations of motion for $\hat{q}^{\alpha}$, which again just give diffeomorphisms, we obtain a gauged nonlinear $\sigma$-model with constrained field strength which proves that the dual geometries are indeed related by a Buscher duality. 


\subsection{T-duality along semichiral isometries $k_{L R}$}

In the presence of semichiral superfields we can no longer decompose the action of the gauged isometry as in the BiLP case (3.7) and separate the rigid piece which acts on the Kähler potential with $\mathcal{L}_{k}$. An extensive treatment of non BiLP geometries is left for [12]. Making the notation of [5] compatible with the previous section we redefine the complex potentials 4 and reduce in the Wess-Zumino gauge:

$$
\begin{aligned}
& \mathbb{V}^{L}\left|=0 \quad, \quad\left(Q_{+} \mathbb{V}^{L}\right)\right|=2 \Gamma_{+} \quad\left(Q_{-} \mathbb{V}^{L}\right) \mid=0, \quad Q_{+} Q_{-} \mathbb{V}^{L}=-2 i\left(\hat{d}^{2}-\hat{d}^{1}\right) \\
& \mathbb{V}^{R}\left|=0 \quad, \quad\left(Q_{+} \mathbb{V}^{R}\right)\right|=0 \quad\left(Q_{-} \mathbb{V}^{R}\right) \mid=2 \Gamma_{-}, \quad Q_{+} Q_{-} \mathbb{V}^{R}=-2 i\left(\hat{d}^{2}+\hat{d}^{1}\right) \\
& \mathbb{V}^{\prime}\left|=0, \quad\left(Q_{+} \mathbb{V}^{\prime}\right)\right|=0 \quad\left(Q_{-} \mathbb{V}^{\prime}\right) \mid=0, \quad Q_{+} Q_{-} \mathbb{V}^{\prime}=-2 i \hat{d}^{3} .
\end{aligned}
$$

The $N=(1,1)$ gauge field-strength $f=i\left(D_{+} \Gamma_{-}+D_{-} \Gamma_{+}\right)$obeys the Bianchi identity

$$
i(\mathbb{F}-\overline{\mathbb{F}}+\tilde{\mathbb{F}}-\overline{\tilde{\mathbb{F}}}) \mid=f
$$

(the $N=(2,2)$ field-strengths $\mathbb{F}, \tilde{\mathbb{F}}$ are given in Appendix $[\mathrm{B})$. Following [5] we write the constrained Lagrange density

$K_{\mathbb{X}}\left(\mathbb{X}_{L}+\overline{\mathbb{X}}_{L}+\mathbb{V}^{L}, \mathbb{X}_{R}+\overline{\mathbb{X}}_{R}+\mathbb{V}^{R}, i\left(\mathbb{X}_{L}-\overline{\mathbb{X}}_{L}+\mathbb{X}_{R}-\overline{\mathbb{X}}_{R}\right)+\mathbb{V}^{\prime}\right)-\tilde{\phi} \mathbb{V}-\overline{\tilde{\phi}} \overline{\mathbb{V}}-\tilde{\chi} \tilde{\mathbb{V}}-\overline{\tilde{\chi}} \overline{\tilde{\mathbb{V}}}$

which reduces to $N=(1,1)$ :

$$
\begin{aligned}
\mathcal{L}=E_{i j} \nabla_{+} \mathbb{X}^{i} \nabla_{-} \mathbb{X}^{j} & +\hat{d}^{1}\left[\left(-i \partial_{L}-i \partial_{\bar{L}}+i \partial_{R}+i \partial_{\bar{R}}\right) K+2(\tilde{\phi}-\overline{\tilde{\phi}})\right] \\
& +\hat{d}^{2}\left[\left(i \partial_{L}+i \partial_{\bar{L}}+i \partial_{R}+i \partial_{\bar{R}}\right) K-2(\tilde{\chi}-\overline{\tilde{\chi}})\right] \\
& +\hat{d}^{3}\left[\frac{1}{2}\left(\partial_{L}-\partial_{\bar{L}}-\partial_{R}+\partial_{\bar{R}}\right) K-i(\tilde{\phi}+\overline{\tilde{\phi}}+\tilde{\chi}+\tilde{\tilde{\chi}})\right] \\
& +f[-i \hat{\phi}-i \overline{\tilde{\phi}}+i \tilde{\chi}+i \overline{\tilde{\chi}}]
\end{aligned}
$$

where $E_{i j}=\left(g_{i j}+B_{i j}\right)$ is the metric and $B$-field of the generalized Kähler geometry as given in, e.g., [14]. As in the previous section, we impose the equations of motion for $\hat{d}^{\alpha}$ to obtain the gauged nonlinear $\sigma$-model with the constraint on the field-strength $f$ that we recognize as the hallmark of T-duality. Again, the $\hat{d}^{\alpha}$ equations of motion just give diffeomorphisms.

\section{Conclusions}

We have used the gauge multiplets constructed in [5, 6] to investigate the duality between semichiral and (twisted) chiral superfields discovered in [1], and found that the dual ge-

\footnotetext{
${ }^{4}$ See Appendix $[$ ] for full details
} 
ometries are related by Buscher duality. We demonstrated this in $N=(2,2)$ superspace where we gave the generalized Kähler potentials with gauged isometries. When we descended to $N=(1,1)$ superspace, the nature of the T-duality was clarified: we found a gauged nonlinear $\sigma$-model with a Lagrange multiplier constraining the field-strength of the gauge field as well as diffeomorphisms relating the generalized moment maps in the original geometry to natural coordinates in the dual geometry.

This work is part of an ongoing exploration of generalized complex geometry, using nonlinear $\sigma$ models, and is therefore complimentary to the mathematical aspects of Tduality considered in [15]. The full construction of the moment maps and a geometric discussion of these results is left for future work [12].

\section{Note:}

After completing our work, we became aware of related results obtained by W. Merrell and D. Vaman.

\section{Acknowledgement:}

UL supported by EU grant (Superstring theory) MRTN-2004-512194 and VR grant 6212006-3365. The work of MR and IR was supported in part by NSF grant no. PHY0354776. The research of R.v.U. was supported by Czech ministry of education contract No. MSM0021622409. The research of M.Z. was supported by VR-grant 621-2004-3177.

\section{A Reduction to $N=(1,1)$ superspace for gauged BiLP geometries}

In this appendix we review some of the results of [5] as they emerge from inherent geometric objects for BiLP geometries. The $N=(1,1)$ invariants system of [5] is slightly modified so that the reduction of the gauged Lagrange density (3.12) to $N=(1,1)$ is simpler in this context; namely, carrying out the reduction for the matter couplings piece will give convenient redefinitions for $N=(1,1)$ gauge invariants. Acting with $Q_{ \pm}$on the gauged action we can identify the connections $A_{ \pm}$that enter with $J_{ \pm} k$ respectively:

$$
\begin{aligned}
Q_{ \pm} K^{(g)}= & K_{i}^{(g)}\left(J_{ \pm j}^{i} D_{ \pm} \varphi^{j}-\frac{1}{4} Q_{ \pm}\left(V^{\phi}+V^{\chi}\right) J_{+j}^{i} k^{j}\right. \\
& \left.-\frac{1}{4} Q_{ \pm}\left(V^{\phi}-V^{\chi}\right) J_{-j}^{i} k^{j}-\frac{1}{4} Q_{ \pm} V^{\prime} \Pi^{i}{ }_{j} k^{j}\right) \\
= & K_{i}^{(g)}\left(J_{ \pm j}^{i} \nabla_{ \pm} \varphi^{j}+\Xi_{ \pm}^{1} J_{\mp j}^{i} k^{j}+\Xi_{ \pm}^{2} \Pi^{i}{ }_{j} k^{j}\right) .
\end{aligned}
$$


We find it useful to modify the $N=(1,1)$ notation of [5], introducing:

$$
\hat{q}^{\phi}=-i \frac{1}{2}\left(Q_{[+} \Xi_{-]}^{1}-D_{[+} \Xi_{-]}^{2}\right) \quad, \quad \hat{q}^{\chi}=-i \frac{1}{2}\left(Q_{(-} \Xi_{+)}^{1}+D_{(+} \Xi_{-)}^{2}\right) \quad, \quad \hat{q}^{\prime}=-i \frac{1}{2} Q_{[+} \Xi_{-]}^{2}
$$

and the field-strength for the connections $A_{ \pm}$

$$
f=-i Q_{(+} \Xi_{-)}^{2}=i\left(D_{+} A_{-}+D_{-} A_{+}\right)
$$

which allows us to write the reduction for $Q_{+} Q_{-} K^{(g)}$ in terms of the geometric objects:

$$
\begin{aligned}
& Q_{+} Q_{-} K^{(g)}= \\
& K_{i j}^{(g)}\left[\left(J_{+k}^{i} \nabla_{+} \varphi^{k}+J_{-k}^{i} k^{k} \Xi_{+}^{1}+\Pi^{i}{ }_{k} k^{k} \Xi_{+}^{2}\right)\left(J_{-l}^{j} \nabla_{-} \varphi^{k}+J_{+l}^{j} k^{l} \Xi_{-}^{1}+\Pi^{j}{ }_{l} k^{l} \Xi_{-}^{2}\right)\right. \\
& \left.\quad-\frac{1}{2}\left(\delta^{i}{ }_{k} \Pi^{j}{ }_{l}+\Pi^{i}{ }_{k} \delta^{j}{ }_{l}\right) \nabla_{+} \varphi^{k} \nabla_{-} \varphi^{l}\right] \\
& \quad+i K_{i}^{(g)} k^{k}\left(\hat{q}^{\phi}\left(J_{+}+J_{-}\right)^{i}{ }_{k}+\hat{q}^{\chi}\left(J_{+}-J_{-}\right)^{i}{ }_{k}+\hat{q}^{\prime} \Pi^{i}{ }_{k}\right)
\end{aligned}
$$

\section{B Conventions and notation}

The conversion between the notation of [5] and the current notation can be derived from changing some signs:

$$
\left\{\mathbb{V}^{\prime}, \mathbb{V}^{R}, V^{\prime}, V^{R}\right\} \rightarrow-\left\{\mathbb{V}^{\prime}, \mathbb{V}^{R}, V^{\prime}, V^{R}\right\}
$$

as well as

$$
\left\{\tilde{\Lambda}, \Lambda^{R}\right\} \rightarrow-\left\{\tilde{\Lambda}, \Lambda^{R}\right\}
$$

These changes correct some unnatural conventions for the definitions of isometries.

We summarize the essential consequences here for both the large vector multiplet and the semichiral vector multiplet in the tables below. 


\begin{tabular}{|c|c|c|}
\hline Object & Old & New \\
\hline $\begin{array}{l}\delta V^{\phi} \\
\delta V^{\chi}\end{array}$ & \multicolumn{2}{|c|}{$\begin{array}{l}i(\bar{\Lambda}-\Lambda) \\
i(\overline{\tilde{\Lambda}}-\tilde{\Lambda})\end{array}$} \\
\hline$\delta V^{\prime}$ & $\Lambda+\bar{\Lambda}+\tilde{\Lambda}+\tilde{\tilde{\Lambda}}$ & $-\Lambda-\bar{\Lambda}+\tilde{\Lambda}+\tilde{\tilde{\Lambda}}$ \\
\hline $\begin{array}{l}\text { Complex potential } \\
\text { and variation (1) }\end{array}$ & $\begin{array}{c}V=\frac{1}{2}\left(V^{\prime}+i\left(V^{\phi}+V^{\chi}\right)\right) \\
\delta V=\Lambda+\tilde{\Lambda}\end{array}$ & $\begin{array}{c}V_{L}=\frac{1}{2}\left(-V^{\prime}+i\left(V^{\phi}-V^{\chi}\right)\right) \\
\delta V_{L}=\Lambda-\tilde{\Lambda}\end{array}$ \\
\hline $\begin{array}{l}\text { Complex potential } \\
\text { and variation }(2)\end{array}$ & $\begin{array}{c}\tilde{V}=\frac{1}{2}\left(V^{\prime}+i\left(V^{\phi}-V^{\chi}\right)\right) \\
\delta V=\Lambda+\overline{\tilde{\Lambda}}\end{array}$ & $\begin{array}{c}V_{R}=\frac{1}{2}\left(-V^{\prime}+i\left(V^{\phi}+V^{\chi}\right)\right) \\
\delta V_{L}=\Lambda-\overline{\tilde{\Lambda}}\end{array}$ \\
\hline $\begin{array}{c}\quad N=(2,2) \\
\text { Gauge invariants }\end{array}$ & $\begin{array}{l}\mathbb{G}_{+}=\overline{\mathbb{D}}_{+} V \\
\mathbb{G}_{-}=\overline{\mathbb{D}}_{-} \tilde{V} \\
\overline{\mathbb{G}}_{+}=\mathbb{D}_{+} \bar{V} \\
\overline{\mathbb{G}}_{-}=\mathbb{D}_{-} \overline{\bar{V}}\end{array}$ & $\begin{array}{l}\mathbb{G}_{+}=\overline{\mathbb{D}}_{+} V_{L} \\
\mathbb{G}_{-}=\overline{\mathbb{D}}_{-} V_{R} \\
\overline{\mathbb{G}}_{+}=\mathbb{D}_{+} \bar{V}_{L} \\
\overline{\mathbb{G}}_{-}=\mathbb{D}_{-} \bar{V}_{R}\end{array}$ \\
\hline $\begin{array}{l}\text { Decomposition } \\
\text { to } N=(1,1)\end{array}$ & \multicolumn{2}{|c|}{$\begin{array}{c}\Xi_{ \pm}^{A}=\left(\operatorname{Re}\left(\mathbb{G}_{ \pm}\right)\left|, \operatorname{Im}\left(\mathbb{G}_{ \pm}\right)\right|\right) \\
D_{ \pm} \Xi_{\mp}^{A}\end{array}$} \\
\hline$q$-invariants: & $\begin{array}{l}\hat{q}^{1}=i\left(Q_{-} \Xi_{+}^{1}-Q_{+} \Xi_{-}^{1}\right) \\
\hat{q}^{2}=i\left(Q_{-} \Xi_{+}^{1}+Q_{+} \Xi_{-}^{1}\right) \\
\hat{q}^{3}=i\left(Q_{-} \Xi_{+}^{2}-Q_{+} \Xi_{-}^{2}\right)\end{array}$ & $\begin{aligned} \hat{q}^{\phi} & =-i \frac{1}{2}\left(Q_{[+} \Xi_{-]}^{1}-D_{[+} \Xi_{-]}^{2}\right) \\
\hat{q}^{\chi} & =-i \frac{1}{2}\left(Q_{(-} \Xi_{+)}^{1}+D_{(+} \Xi_{-)}^{2}\right) \\
\hat{q}^{\prime} & =-i \frac{1}{2}\left(Q_{+} \Xi_{-}^{2}-Q_{-} \Xi_{+}^{2}\right)\end{aligned}$ \\
\hline The field-strength $f$ & $i\left(Q_{+} \Xi_{-}^{2}+Q_{-} \Xi_{+}^{2}\right)$ & $-i\left(Q_{+} \Xi_{-}^{2}+Q_{-} \Xi_{+}^{2}\right)$ \\
\hline
\end{tabular}

Table 1: Large vector multiplet conventions and definitions 


\begin{tabular}{|c|c|c|}
\hline Object & Old & New \\
\hline $\begin{array}{l}\delta \mathbb{V}^{L} \\
\delta \mathbb{V}^{R}\end{array}$ & \multicolumn{2}{|c|}{$\begin{array}{l}i\left(\bar{\Lambda}_{L}-\Lambda_{L}\right) \\
i\left(\bar{\Lambda}_{R}-\Lambda_{R}\right)\end{array}$} \\
\hline$\delta \mathbb{V}^{\prime}$ & $\Lambda_{L}+\bar{\Lambda}_{L}+\Lambda_{R}+\bar{\Lambda}_{R}$ & $-\Lambda_{L}-\bar{\Lambda}_{L}+\Lambda_{R}+\bar{\Lambda}_{R}$ \\
\hline $\begin{array}{l}\text { Complex potential } \\
\text { and variation (1) }\end{array}$ & $\begin{array}{c}\mathbb{V}=\frac{1}{2}\left(\mathbb{V}^{\prime}+i\left(\mathbb{V}^{L}+\mathbb{V}^{R}\right)\right) \\
\delta \mathbb{V}=\Lambda_{L}+\Lambda_{R}\end{array}$ & $\begin{array}{c}\mathbb{V}=\frac{1}{2}\left(-\mathbb{V}^{\prime}+i\left(\mathbb{V}^{L}-\mathbb{V}^{R}\right)\right) \\
\delta \mathbb{V}=\Lambda_{L}-\Lambda_{R}\end{array}$ \\
\hline $\begin{array}{l}\text { Complex potential } \\
\text { and variation }(2)\end{array}$ & $\begin{array}{c}\tilde{\mathbb{V}}=\frac{1}{2}\left(\mathbb{V}^{\prime}+i\left(\mathbb{V}^{L}-\mathbb{V}^{R}\right)\right) \\
\delta \mathbb{V}=\Lambda_{L}+\bar{\Lambda}_{R}\end{array}$ & $\begin{array}{c}\tilde{\mathbb{V}}=\frac{1}{2}\left(-\mathbb{V}^{\prime}+i\left(\mathbb{V}^{L}+\mathbb{V}^{R}\right)\right) \\
\delta \mathbb{V}=\Lambda_{L}-\bar{\Lambda}_{R}\end{array}$ \\
\hline $\begin{array}{l}\quad N=(2,2) \\
\text { Gauge invariants }\end{array}$ & $\begin{array}{l}\mathbb{F}=\overline{\mathbb{D}}_{+} \overline{\mathbb{D}}_{-} \mathbb{V} \\
\tilde{\mathbb{F}}=\overline{\mathbb{D}}_{+} \mathbb{D}_{-} \tilde{\mathbb{V}}\end{array}$ & $\begin{array}{l}\overline{\mathbb{F}}=-\mathbb{D}_{+} \mathbb{D}_{-} \overline{\mathbb{V}} \\
\overline{\tilde{\mathbb{F}}}=-\mathbb{D}_{+} \overline{\mathbb{D}}{ }_{-} \overline{\tilde{V}}\end{array}$ \\
\hline$\hat{d}$-invariants & $\hat{d}^{1}=(\mathbb{F}+\overline{\mathbb{F}}) \mid, \quad \hat{d}^{2}=(\tilde{\mathbb{F}}+$ & $\mid, \quad \hat{d}^{3}=i(\mathbb{F}-\overline{\mathbb{F}}-\tilde{\mathbb{F}}+\overline{\tilde{\mathbb{F}}})$ \\
\hline Gauge fields & $\begin{array}{c}\Gamma_{+}=\frac{1}{2}\left(Q_{+} \mathbb{V}^{L}-\frac{1}{2} D_{+} \mathbb{V}^{\prime}\right) \mid \\
\Gamma_{-}=-\frac{1}{2}\left(Q_{-} \mathbb{V}^{R}-\frac{1}{2} D_{-} \mathbb{V}^{\prime}\right) \mid\end{array}$ & $\begin{array}{l}\Gamma_{+}=\frac{1}{2}\left(Q_{+} \mathbb{V}^{L}+\frac{1}{2} D_{+} \mathbb{V}^{\prime}\right) \mid \\
\Gamma_{-}=\frac{1}{2}\left(Q_{-} \mathbb{V}^{R}-\frac{1}{2} D_{-} \mathbb{V}^{\prime}\right) \mid\end{array}$ \\
\hline Bianchi identity & $i(\mathbb{F}-\overline{\mathbb{F}}+\tilde{\mathbb{F}}-\tilde{\tilde{\mathbb{F}}}) \mid=$ & $f=i\left(D_{+} \Gamma_{-}+D_{-} \Gamma_{+}\right)$ \\
\hline
\end{tabular}

Table 2: Semichiral vector multiplet conventions and definitions 


\section{References}

[1] M. T. Grisaru, M. Massar, A. Sevrin and J. Troost, "Some aspects of N $=(2,2)$, D = 2 supersymmetry," Fortsch. Phys. 47, 301 (1999) arXiv:hep-th/9801080.

[2] J. Bogaerts, A. Sevrin, S. van der Loo and S. Van Gils, "Properties of semichiral superfields," Nucl. Phys. B562 (1999) 277 arXiv:hep-th/9905141].

[3] N. J. Hitchin, A. Karlhede, U. Lindström and M. Roček, "Hyperkähler Metrics And Supersymmetry," Commun. Math. Phys. 108, 535 (1987).

[4] M. Roček and E. P. Verlinde, "Duality, quotients, and currents," Nucl. Phys. B 373, 630 (1992) arXiv:hep-th/9110053.

[5] U. Lindstrom, M. Roček, I. Ryb, R. von Unge and M. Zabzine, "New N = $(2,2)$ vector multiplets," arXiv:0705.3201 [hep-th].

[6] S. J. J. Gates and W. Merrell, " $\mathrm{D}=2 \mathrm{~N}=(2,2)$ Semi Chiral Vector Multiplet," arXiv:0705.3207 [hep-th].

[7] S. J. Gates, C. M. Hull and M. Roček, "Twisted Multiplets And New Supersymmetric Nonlinear Sigma Models," Nucl. Phys. B248 (1984) 157.

P. S. Howe and G. Sierra, "Two-Dimensional Supersymmetric Nonlinear Sigma Models With Torsion," Phys. Lett. B148, 451 (1984).

T. Buscher, U. Lindström and M. Roček, "New Supersymmetric Sigma Models With Wess-Zumino Terms," Phys. Lett. B202, 94 (1988).

C. M. Hull and B. J. Spence, "The gauged nonlinear sigma-model with WessZumino term," Phys. Lett. B 232 (1989) 204; "The Geometry of the gauged sigma-model with Wess-Zumino term," Nucl. Phys. B 353 (1991) 379.

M. Roček, K. Schoutens and A. Sevrin, "Off-Shell WZW Models In Extended Superspace," Phys. Lett. B 265, 303 (1991).

I. T. Ivanov, B. B. Kim and M. Roček, "Complex structures, duality and WZW models in extended superspace," Phys. Lett. B343 (1995) 133 arXiv:hep-th/9406063|.

S. Lyakhovich and M. Zabzine, "Poisson geometry of sigma models with extended supersymmetry," Phys. Lett. B548 (2002) 243 [arXiv:hep-th/0210043].

N. Hitchin, "Generalized Calabi-Yau manifolds," Q. J. Math. 54 (2003), no. 3, 281 308, arXiv:math.DG/0209099|. 
G. Lopes Cardoso, G. Curio, G. Dall'Agata and D. Lust, "Heterotic string theory on non-Kaehler manifolds with H-flux and gaugino condensate," Fortsch. Phys. 52, 483 (2004) arXiv:hep-th/0310021.

A. Kapustin and Y. Li, "Topological sigma-models with H-flux and twisted generalized complex manifolds," arXiv:hep-th/0407249.

S. Chiantese, F. Gmeiner and C. Jeschek, "Mirror symmetry for topological sigma models with generalized Kaehler geometry," Int. J. Mod. Phys. A 21, 2377 (2006) arXiv:hep-th/0408169.

R. Zucchini, "A sigma model field theoretic realization of Hitchin's generalized complex geometry," JHEP 0411 (2004) 045 arXiv:hep-th/0409181.

"A topological sigma model of biKaehler geometry," JHEP 0601, 041 (2006)

M. Gualtieri, "Generalized complex geometry," Oxford University DPhil thesis, arXiv:math.DG/0401221. arXiv:hep-th/0511144.

"The biHermitian topological sigma model," JHEP 0612, 039 (2006) arXiv:hep-th/0608145.

"BiHermitian supersymmetric quantum mechanics," Class. Quant. Grav. 24, 2073 (2007) arXiv:hep-th/0611308.

L. Bergamin, "Generalized complex geometry and the Poisson sigma model," Mod. Phys. Lett. A 20, 985 (2005) arXiv:hep-th/0409283.

U. Lindström, "Generalized $\mathrm{N}=(2,2)$ supersymmetric non-linear sigma models," Phys. Lett. B 587, 216 (2004) arXiv:hep-th/0401100.

U. Lindström, R. Minasian, A. Tomasiello and M. Zabzine, "Generalized complex manifolds and supersymmetry," Commun. Math. Phys. 257, 235 (2005) arXiv:hep-th/0405085.

M. Zabzine, "Hamiltonian perspective on generalized complex structure," Commun. Math. Phys. 263 (2006) 711 arXiv:hep-th/0502137.

N. Hitchin, "Instantons, Poisson structures and generalized Kähler geometry," arXiv:math.DG/0503432.

M. Grana, J. Louis and D. Waldram, "Hitchin functionals in N = 2 supergravity," JHEP 0601, 008 (2006) arXiv:hep-th/0505264.

P. Koerber, "Stable D-branes, calibrations and generalized Calabi-Yau geometry," JHEP 0508, 099 (2005) arXiv:hep-th/0506154.

Y. Li, "On deformations of generalized complex structures: The generalized Calabi-Yau case," arXiv:hep-th/0508030.

A. Bredthauer, U. Lindström and J. Persson, "First-order supersymmet- 
ric sigma models and target space geometry," JHEP 0601, 144 (2006) arXiv:hep-th/0508228.

$\mathrm{S} . \mathrm{Hu}$, "Hamiltonian symmetries and reduction in generalized geometry," arXiv:math.DG/0509060.

H. Bursztyn, G. Cavalcanti and M. Gualtieri, "Reduction of Courant algebroids and generalized complex structures," arXiv:math.DG/0509640

Y. Lin and S. Tolman, "Reduction of twisted generalized Kähler structure," arXiv:math.DG/0510010

I. Calvo, "Supersymmetric WZ-Poisson sigma model and twisted generalized complex geometry," arXiv:hep-th/0511179.

V. Pestun, "Topological strings in generalized complex space," arXiv:hep-th/0603145.

M. Zabzine, "Lectures on generalized complex geometry and supersymmetry," arXiv:hep-th/0605148.

W. y. Chuang, "Topological twisted sigma model with H-flux revisited," arXiv:hep-th/0608119.

W. Merrell, L. A. P. Zayas and D. Vaman, "Gauged $(2,2)$ sigma models and generalized Kaehler geometry," arXiv:hep-th/0610116.]

A. Kapustin and A. Tomasiello, "The general $(2,2)$ gauged sigma model with three-form flux," arXiv:hep-th/0610210.

[8] T. H. Buscher, "A Symmetry of the String Background Field Equations," Phys. Lett. B 194, 59 (1987). "Path Integral Derivation of Quantum Duality in Nonlinear Sigma Models," Phys. Lett. B 201, 466 (1988).

[9] S. J. Gates, C. M. Hull and M. Roček, "Twisted Multiplets And New Supersymmetric Nonlinear Sigma Models," Nucl. Phys. B248 (1984) 157.

[10] T. Buscher, U. Lindström and M. Roček, "New Supersymmetric $\sigma$-models with Wess-Zumino terms," Phys. Lett. B 202, 94 (1988).

[11] C. M. Hull, A. Karlhede, U. Lindström and M. Roček, "Nonlinear Sigma Models And Their Gauging In And Out Of Superspace," Nucl. Phys. B 266, 1 (1986).

[12] U. Lindström, M. Roček, I. Ryb, R. von Unge and M. Zabzine, to appear.

[13] U. Lindström, M. Roček, R. von Unge and M. Zabzine, "Linearizing Generalized Kahler Geometry," JHEP 0704, 061 (2007) arXiv:hep-th/0702126. 
[14] U. Lindström, M. Roček, R. von Unge and M. Zabzine, "Generalized Kähler manifolds and off-shell supersymmetry," Commun. Math. Phys. 269, 833 (2007) arXiv:hep-th/0512164].

[15] G. Cavalcanti, "New aspects of the ddc-lemma," Oxford University D. Phil thesis, arXiv:math/0501406. 